\title{
Reduced C9orf72 gene expression in c9FTD/ALS is caused by histone trimethylation, an epigenetic event detectable in blood
}

\author{
Veronique V. Belzil • Peter O. Bauer · Mercedes Prudencio · Tania F. Gendron · Caroline T. Stetler · Irene K. Yan • \\ Luc Pregent • Lillian Daughrity • Matthew C. Baker · Rosa Rademakers · Kevin Boylan · Tushar C. Patel • \\ Dennis W. Dickson $\cdot$ Leonard Petrucelli
}

Received: 1 July 2013 / Accepted: 16 October 2013 / Published online: 29 October 2013

(C) The Author(s) 2013. This article is published with open access at Springerlink.com

\begin{abstract}
Individuals carrying (GGGGCC) expanded repeats in the C9orf72 gene represent a significant portion of patients suffering from amyotrophic lateral sclerosis (ALS) and frontotemporal dementia (FTD). Elucidating how these expanded repeats cause "c9FTD/ALS" has since become an important goal of the field. Toward this end, we sought to investigate whether epigenetic changes are responsible for the decrease in C9orf72 expression levels observed in c9FTD/ALS patients. We obtained brain tissue from ten c9FTD/ALS individuals, nine FTD/ALS cases without a $C 9$ orf72 repeat expansion, and nine disease control participants, and generated fibroblastoid cell lines from seven C9orf72 expanded repeat carriers and seven participants carrying normal alleles. Chromatin
\end{abstract}

V. V. Belzil and P. O. Bauer contributed equally to this work.

Electronic supplementary material The online version of this article (doi:10.1007/s00401-013-1199-1) contains supplementary material, which is available to authorized users.

V. V. Belzil · P. O. Bauer · M. Prudencio · T. F. Gendron ·

C. T. Stetler $\cdot$ L. Pregent $\cdot$ L. Daughrity $\cdot$ M. C. Baker .

R. Rademakers · D. W. Dickson · L. Petrucelli $(\bowtie)$

Department of Research, Neuroscience, Mayo Clinic College

of Medicine, 4500 San Pablo Road, Jacksonville, FL 32224, USA

e-mail: petrucelli.leonard@mayo.edu

I. K. Yan

Department of Cancer Biology, Mayo Clinic College

of Medicine, 4500 San Pablo Road, Jacksonville, FL 32224, USA

K. Boylan

Department of Neurology, Mayo Clinic, 4500 San Pablo Road,

Jacksonville, FL 32224, USA

T. C. Patel

Department of Transplantation, Mayo Clinic, 4500 San Pablo

Road, Jacksonville, FL 32224, USA immunoprecipitation using antibodies for histone $\mathrm{H} 3$ and H4 trimethylated at lysines 9 (H3K9), 27 (H3K27), 79 (H3K79), and 20 (H4K20) revealed that these trimethylated residues bind strongly to $C 9$ orf 72 expanded repeats in brain tissue, but not to non-pathogenic repeats. Our finding that $C 9$ orf 72 mRNA levels are reduced in the frontal cortices and cerebella of c9FTD/ALS patients is consistent with trimethylation of these histone residues, an event known to repress gene expression. Moreover, treating repeat carrier-derived fibroblasts with 5-aza-2-deoxycytidine, a DNA and histone demethylating agent, not only decreased C9orf72 binding to trimethylated histone residues, but also increased $C 9$ orf 72 mRNA expression. Our results provide compelling evidence that trimethylation of lysine residues within histones $\mathrm{H} 3$ and $\mathrm{H} 4$ is a novel mechanism involved in reducing C9orf72 mRNA expression in expanded repeat carriers. Of importance, we show that mutant $C 9$ orf 72 binding to trimethylated $\mathrm{H} 3 \mathrm{~K} 9$ and $\mathrm{H} 3 \mathrm{~K} 27$ is detectable in blood of c9FTD/ALS patients. Confirming these exciting results using blood from a larger cohort of patients may establish this novel epigenetic event as a biomarker for c9FTD/ALS.

Keywords Amyotrophic lateral sclerosis .

Frontotemporal dementia $\cdot$ C9orf72 Epigenetic modification $\cdot$ Repeat expansion $\cdot$ Histone methylation

\section{Introduction}

Amyotrophic lateral sclerosis (ALS) and frontotemporal dementia (FTD) are two devastating neurodegenerative diseases seen in comorbidity in up to $50 \%$ of patients affected with either disease [23, 28]. ALS, the most frequently occurring motor neuron disease [26], is characterized by 
degeneration of upper and lower motor neurons leading to muscle weakness, spasticity and atrophy during midadulthood, with patients having a mean life expectancy of 3-5 years after disease onset [7]. Frontotemporal dementia is one of the most frequent presenile onset dementias [19], with neuronal degeneration in the frontal and temporal lobes causing a progressive deterioration of behavior, personality and/or language [24]. Recent findings demonstrate that an expanded non-coding hexanucleotide (GGGGCC) repeat in the chromosome 9 open reading frame 72 gene (C9orf72, OMIM *614260) is the most common known cause of FTD and ALS, collectively referred to as c9FTD/ALS [16, 36]. Mutation carriers have several hundred to several thousand copies of the (GGGGCC) hexanucleotide repeat, as compared to 30 or less copies in nonpathogenic carriers [16]. This discovery provided further evidence that the two diseases result from defects in the same or overlapping biological pathways.

Recent studies have suggested that C9orf72 repeat expansions may lead to RNA-mediated toxicity through the formation of RNA foci that possibly sequester RNA-binding proteins, as well as repeat associated non-ATG (RAN) translation leading to the synthesis of aggregation-prone c9RAN proteins $[2,16,30]$. Decreases in C9orf72 mRNA levels in brain and lymphoblasts of repeat expansion carriers have also been reported [16, 22, 36], indicating that loss of C9orf72 function may also play a role in disease pathogenesis. Moreover, the finding that downregulation of the zebrafish orthologue of C9orf72 leads to both altered morphology of motor neuron axons and locomotor deficits, and that these features are reversed upon overexpression of wild-type human $C 9$ orf 72 , further support the notion that C9orf72 loss of function is detrimental [14]. Dual involvement of RNA toxicity and loss of function have been implicated in a variety of neurodegenerative diseases caused by repeat expansions, including myotonic dystrophy, spinocerebellar ataxia (SCA) types 8 and 31, and Fragile X-associated tremor/ataxia syndromes [35].

Increasing evidence suggests that repeat expansions can impair mRNA expression through epigenetic changes resulting from variation in DNA and histone methylation, which lead to aberrant binding of the transcriptional machinery as a consequence of heterochromatin formation [1, 4, 15, 27, 32, 41]. Epigenetic changes and altered mRNA expression have previously been linked to FTD/ALS pathogenesis. For instance, aberrant epigenetic regulation of granulin $(G R N)$, the gene that encodes progranulin (PGRN) and is mutated in about $5-10 \%$ of the total FTD population, causes a decrease in GRN mRNA expression [18]. Of interest, protein levels of DNA methyltransferases (DNMTs), which induce DNA methylation by catalyzing methyl group transfer to cytosine residues in regulatory regions, increase pre-apoptotically in mouse motor neurons [13], and DNMT1, DNMT3a, and 5-methylcytosine are all upregulated in motor neurons of ALS patients [13]. DNMT1 has been reported to function as a maintenance methyltransferase both in repeat regions and repetitive genomic sequences characterized by a higher frequency of cytosine-guanine sequences, known as cytosine-phosphate-guanine $(\mathrm{CpG})$ islands, whereas DNMT3 mainly functions in $\mathrm{CpG}$ islands. These islands, which are normally not methylated in active genes, are usually methylated to suppress gene expression of imprinted genes [9]. An observed-to-expected $\mathrm{CpG}$ ratio greater than $60 \%$ predicts $\mathrm{CpG}$ islands in a genomic DNA region larger than $200 \mathrm{bp}$ [20]. Based on this criterion, the observed-to-expected $\mathrm{CpG}$ ratio is $75 \%$ in patients carrying 700-1,600 (GGGGCC) copies. This suggests the presence of a newly formed $\mathrm{CpG}$ island spanning the repeat region that is potentially at risk of abnormal methylation in expansion carriers. Given that methylation of repeat sequences, as well as hypermethylation of adjacent $\mathrm{CpG}$ islands within promoter regions, has been shown to aberrantly influence histone methylation processes leading to gene silencing and consequently loss of protein function $[1,37]$, we sought to determine whether a similar disease cascade occurs in c9FTD/ALS.

To elucidate the mechanisms causing decreased expression of $C 9$ orf72 mRNA in mutation carriers [16, 22, 36], which could lead to loss of C9orf72 function, we investigated whether perturbed epigenetic processes are involved. Our results clearly demonstrate that trimethylation of histones $\mathrm{H} 3$ and $\mathrm{H} 4$ at several lysine residues is a novel mechanism involved in decreasing the expression of $\mathrm{C} 9 \mathrm{orf} 72$ mRNA in expanded repeat carriers. In addition, using peripheral blood of two expanded repeat carriers, we confirmed that these epigenetic changes are easily detectable. Future studies will need to confirm blood detection of epigenetic changes in a larger cohort of c9FTD/ALS patients to validate the biomarker capacity of this novel epigenetic event.

\section{Materials and methods}

Standard protocol approvals, registrations, and patient consents

Protocols were approved by the Mayo Clinic Institutional Review Board and Ethics Committee on human experimentation. All participants or authorized family members gave written informed consent after which participant and family information was gathered, skin biopsies were collected, or post-mortem analyses were performed. 
Subjects

All participants in this study were recruited at Mayo Clinic Florida and were independently ascertained as having ALS and/or FTD by trained neurologists. Frontal cortices and cerebella tissues were obtained after post-mortem analyses from four ALS and six FTD patients carrying the C9orf 72 expansion, four ALS and five FTD cases without $C 9$ orf 72 expansion, and nine disease control participants (clinical information in online resource, Table 2). Skin biopsies were collected from a total of seven C9orf 72 expansion carriers, including two healthy carriers of 28 and 30 years of age, and seven participants carrying normal alleles (clinical information in online resource, Table 3). Blood samples were obtained from two ALS patients carrying the repeat expansion, and two ALS cases carrying normal alleles (clinical information in online resource, Table 4).

\section{Maintenance and treatment of fibroblasts}

Fibroblasts were derived from skin sampled by punch biopsy on the anterior aspect of the forearm. Fibroblasts were maintained in Dulbecco's modified Eagle's medium (Lonza, Basel, Switzerland) supplemented with $10 \%$ heatinactivated fetal bovine serum (Sigma-Aldrich, St-Louis, MO, USA), 100 units $/ \mathrm{ml}$ penicillin, and $100 \mu \mathrm{g} / \mathrm{ml}$ streptomycin (Gibco, Carlsbad, CA, USA) at $37^{\circ} \mathrm{C}$, in an atmosphere containing $5 \% \mathrm{CO}_{2}$ and $95 \%$ air. Cells were treated with $2 \mu \mathrm{M}$ 5-aza-2-deoxycytidine (5-AZA) or dimethyl sulfoxide (DMSO) for 6 days before they were harvested for further experiments.

Quantitative real-time polymerase chain reaction (qRT-PCR)

Total RNA was extracted from fibroblasts using Trizol (Invitrogen, Carlsbad, CA, USA) and from brain tissue using the RNAeasy Plus Micro Kit (QIAGEN, Venlo, Limberg, Netherlands) as per manufacturer's instructions. RNA integrity was verified on an Agilent 2100 bioanalyzer (Agilent Technologies, Santa Clara, CA, USA). cDNA was obtained after reverse transcription polymerase chain reactions (RT-PCR) using approximately $1 \mu \mathrm{g}$ of RNA with random primers and the High Capacity cDNA Transcription Kit (Applied Biosystems, Foster City, CA, USA) as per manufacturer's instructions. Following standard protocols, qRT-PCR was conducted in triplicates for all samples using inventoried TaqMan gene expression assays for C9orf72 transcript variants 1 (NM_145005.5), 2 (NM_018325.3) and 3 (NM_001256054.1) (Hs00376619), C9orf72 transcript variants 2 and 3 (Hs00945132), C9orf72 transcript variant 1 (Hs00331877), GAPDH (Hs00266705), and H19 (Hs 00262142) (Applied Biosystems) on an ABI Prism
7900HT Fast Real-Time PCR System (Applied Biosystems). Relative quantification was determined using the $\triangle \triangle \mathrm{Ct}$ method and normalized to $G A P D H$.

\section{Droplet digital PCR (ddPCR)}

RNA was isolated from brain tissue using the RNAeasy Plus Micro Kit (QIAGEN) and cDNA was obtained by reverse transcription using random primers and the High Capacity cDNA Transcription Kit (Applied Biosystems) as per manufacturer's instructions. Reaction mixtures were prepared for droplet digital PCR using $4 \mu 1$ of reverse transcribed product, $10 \mu \mathrm{l}$ of ddPCR $2 \mathrm{x}$ Master Mix (Bio-Rad, Hercules, CA, USA), $1 \mu 1$ of 20x Primer and TaqMan Probe assays for C9orf72 transcript variant 1, 2 and 3 (Hs00376619), C9orf72 transcript variant 2 and 3 (Hs00945132), C9orf72 transcript variant 1 (Hs00331877) (Applied Biosystems), and $5 \mu 1$ of nuclease-free water, per reaction mixture. The negative control contained water only. Emulsified $1 \mathrm{~nL}$ reaction droplets were generated using a QX100 Droplet generator (Bio-Rad) and a droplet generator DG8 cartridge (Bio-Rad) containing $20 \mu \mathrm{l}$ of reaction mixture and $70 \mu 1$ of ddPCR droplet generation oil (Bio-Rad) per well. Thirty-five microliters of the generated droplet emulsions were transferred to 96-well PCR plates which were heat-sealed using foil sheets. Target DNA amplification was performed by thermal cycling the droplet emulsions as follows: initial denaturation at $95{ }^{\circ} \mathrm{C}$ for $10 \mathrm{~min}$, followed by 40 cycles of $94{ }^{\circ} \mathrm{C}$ for $30 \mathrm{~s}$ and $60{ }^{\circ} \mathrm{C}$ for $1 \mathrm{~min}$, then $98^{\circ} \mathrm{C}$ for $10 \mathrm{~min}$. The fluorescence of each thermal cycled droplet was measured using the QX100 droplet reader (Bio-Rad). Data was analyzed using the QuantaSoft software (Bio-Rad) after threshold setting on fluorescence of negative controls.

\section{Chromatin immunoprecipitation (ChIP)}

Fibroblasts grown in $10 \mathrm{~cm}$ culture plates at $90-95 \%$ confluency were treated with DMSO or 5-AZA followed by incubation with $1 \%$ formaldehyde at $37{ }^{\circ} \mathrm{C}$ for $10 \mathrm{~min}$ for crosslinking. Glycine (125 $\mathrm{mM})$ was then added and cells were incubated at room temperature for $5 \mathrm{~min}$ to quench the crosslinking. Fibroblasts were washed with ice cold PBS, scraped and pelleted by brief centrifugation at $13,000 \times g$. Cell pellets were resuspended in $500 \mu 1$ SDS lysis buffer (EMD Millipore, Billerica, MA, USA) containing protease inhibitors and incubated on ice for $10 \mathrm{~min}$. DNA was sheared by four $10 \mathrm{~s}-\mathrm{on} / 30 \mathrm{~s}$-off cycles of sonication at $35 \%$ output. $100 \mu \mathrm{l}$ of each sample was then diluted with $900 \mu \mathrm{l}$ ChIP dilution buffer (EMD Millipore). For pre-cleaning, the samples were incubated with $40 \mu \mathrm{l}$ of Protein A Agarose slurry (EMD Millipore) with rotation for $1 \mathrm{~h}$ at $4{ }^{\circ} \mathrm{C}$. Samples were then incubated overnight at 
$4{ }^{\circ} \mathrm{C}$ with $2 \mu \mathrm{g}$ of anti-(pan)H3 (EMD Millipore) or antiH3K9me3 (Diagenode, Denville, NJ, USA). ChIP from brain samples was performed using $50-70 \mathrm{mg}$ of tissue homogenized using a tissue grinder after crosslinking and quenching, and following the same procedure used for fibroblasts with the exception that samples were incubated overnight at $4{ }^{\circ} \mathrm{C}$ with $2 \mu \mathrm{g}$ of anti-(pan)H3 and $\mathrm{H} 4$ (EMD Millipore), as well as anti-H3K9me3, H3K27me3, H3K79me3, H4K20me3 (Diagenode). Overnight incubation with antibodies was followed by a $3 \mathrm{~h}$ incubation with $50 \mu \mathrm{l}$ agarose slurry at $4{ }^{\circ} \mathrm{C}$. ChIP from blood was performed using mononuclear cells isolated using standard conditions, and washed twice using HANK'S solution (Life Technologies, Carlsbad, CA, USA). DNA was sheared by six $10 \mathrm{~s}$-on/30 s-off cycles of sonication at $30 \%$ output, then subjected to the same ChIP procedure as used for fibroblast studies. However, overnight incubation at $4{ }^{\circ} \mathrm{C}$ was performed using $1 \mu \mathrm{g}$ of anti-(pan)H3 (EMD Millipore), anti-H3K9me3, and anti-H3K27me3 (Diagenode) antibodies followed by a $3 \mathrm{~h}$ incubation with $25 \mu \mathrm{l}$ agarose slurry at $4{ }^{\circ} \mathrm{C}$. The bound complexes for the three experiments were washed for $10 \mathrm{~min}$ at room temperature with rotation, first with low salt immune complex wash buffer, followed by high salt and $\mathrm{LiCl}$ immune complex wash buffers (all from EMD Millipore), and two final washes with TE buffer. After the final wash with TE buffer, the agarose was incubated with freshly prepared ChIP elution buffer $\left(0.1 \mathrm{M} \mathrm{NaHCO}_{3}, 1 \% \mathrm{SDS}\right)$ for 20 min at room temperature with rotation, after which the agarose beads were discarded. After reverse crosslinking with $200 \mathrm{mM} \mathrm{NaCl}$ at $65{ }^{\circ} \mathrm{C}$ either overnight for the fibroblast and brain studies, or $6 \mathrm{~h}$ for the blood study, samples were treated with $0.5 \mu \mathrm{g} / \mu 1$ Proteinase $\mathrm{K}$ for $90 \mathrm{~min}$ at $45{ }^{\circ} \mathrm{C}$. The eluted DNA was purified using a DNA Cleanup kit (Zymo Research, Irvine, CA, USA) for the analysis. For semi-quantitative (semi-q) PCR, forward primer 5'AGAGGGTGGGAAAAACAAAAACAC3' and reverse primer 5'AAAACCACGAAATCGTCTTCACTT3' were designed using PrimerSelect (DNASTAR, Madison, WI, USA). DNA amplification was conducted by PCR using $1 \mu \mathrm{l}$ of ChIP-obtained DNA, $0.75 \mu \mathrm{l} \mathrm{dNTP}, 1.5 \mu \mathrm{l} 10 \mathrm{x}$ standard buffer (including $1.5 \mathrm{mM}$ final $\mathrm{MgCl}_{2}$ concentration), $0.6 \mu \mathrm{l}$ of each primer, $10.25 \mu \mathrm{l}$ of nuclease-free water, and $0.3 \mu l$ of Apex Taq Polymerase (Genesee Scientific) per reaction mixture, and was run on a Mastercycler proS (Eppendorf). For each fragment, a denaturation step of 5 min was first performed at $95{ }^{\circ} \mathrm{C}$. After that, a touchdown protocol was used which consisted of an initial cycle of $20 \mathrm{~s}$ denaturation at $94{ }^{\circ} \mathrm{C}, 20 \mathrm{~s}$ annealing at $65^{\circ} \mathrm{C}$ and $30 \mathrm{~s}$ elongation at $72{ }^{\circ} \mathrm{C}$. This was followed by ten cycles in which the annealing temperature was decreased each time by $1{ }^{\circ} \mathrm{C}$. Then 40 cycles of $20 \mathrm{~s}$ denaturation at $94{ }^{\circ} \mathrm{C}$, $20 \mathrm{~s}$ annealing at $58{ }^{\circ} \mathrm{C}$ and $30 \mathrm{~s}$ elongation at $72{ }^{\circ} \mathrm{C}$ were run. A final extension at $72{ }^{\circ} \mathrm{C}$ was performed for $5 \mathrm{~min}$. DNA products were electrophoresed in a $2 \%$ agarose gel.

Statistical analysis

We used unpaired Student's $t$ test for comparison between two sample groups with a $95 \%$ confidence level. One-way ANOVA followed by Tukey post hoc test was used for multiple comparisons with a $95 \%$ confidence level. We considered the difference between comparisons to be significant when $p<0.05(* p<0.05 ; * * p<0.01 ; * * *<0.005)$.

\section{Results}

Given that brain tissue analysis of $C 9$ orf 72 mRNA expression levels has been limited to the frontal cortex [16, 22], we first sought to explore whether C9orf72 mRNA expression is similarly decreased in the frontal cortex of our cohort of patients, and to evaluate whether a reduction is also observed in the cerebellum, another brain region affected in c9FTD/ALS [34]. We obtained brain tissue samples from ten ALS and FTD C9orf72 repeat carriers (C9orf72+), nine ALS and FTD patients carrying a non-pathogenic C9orf72 repeat (C9orf72-), and nine C9orf72- disease controls (clinical information in online resource, Table 2). We then isolated RNA from cerebella and frontal cortices and performed expression assays by quantitative real-time polymerase chain reaction (qRTPCR) using Taqman probes targeting transcript variants 1 , 2 and 3, as well as transcript variants 2 and 3 (Fig. 1a). As transcript variant 1 was difficult to detect through standard qRT-PCR, we assessed its expression using a highly precise and absolute nucleic acid quantification technique, termed droplet digital PCR (ddPCR) [25]. Moreover, we further compared expression levels obtained from standard qRTPCR with ddPCR (online resource, Fig. 1, Table 1). We found that the C9orf72+ group exhibited decreased mRNA expression across all assays, as compared to normal repeat length carriers and disease control participants (Fig. 1b-e).

We next explored whether this decrease in C9orf72 expression results from aberrant histone methylation, given that trimethylation of histone $\mathrm{H} 3$ at lysines 9, 27, 79 , as well as of histone $\mathrm{H} 4$ at lysine 20 , is linked to gene repression [3]. Using brain tissue from all 28 participants, we performed chromatin immunoprecipitation (ChIP) experiments using anti-H3K9me3, anti-H3K27me3, anti$\mathrm{H} 3 \mathrm{~K} 79$ me3, anti-(pan)H3 (total histone $\mathrm{H} 3$ ), as well as anti-H4K20me3 and anti-(pan)H4 (total histone H4). We successfully amplified the C9orf72 promoter region in pathogenic repeat carriers, and found that the trimethylated residues only bind strongly to $C 9$ orf 72 repeat expansions (Fig. 2a-e). Of note, the C9orf72 genomic region 
a c9orf 72 transcript variants:
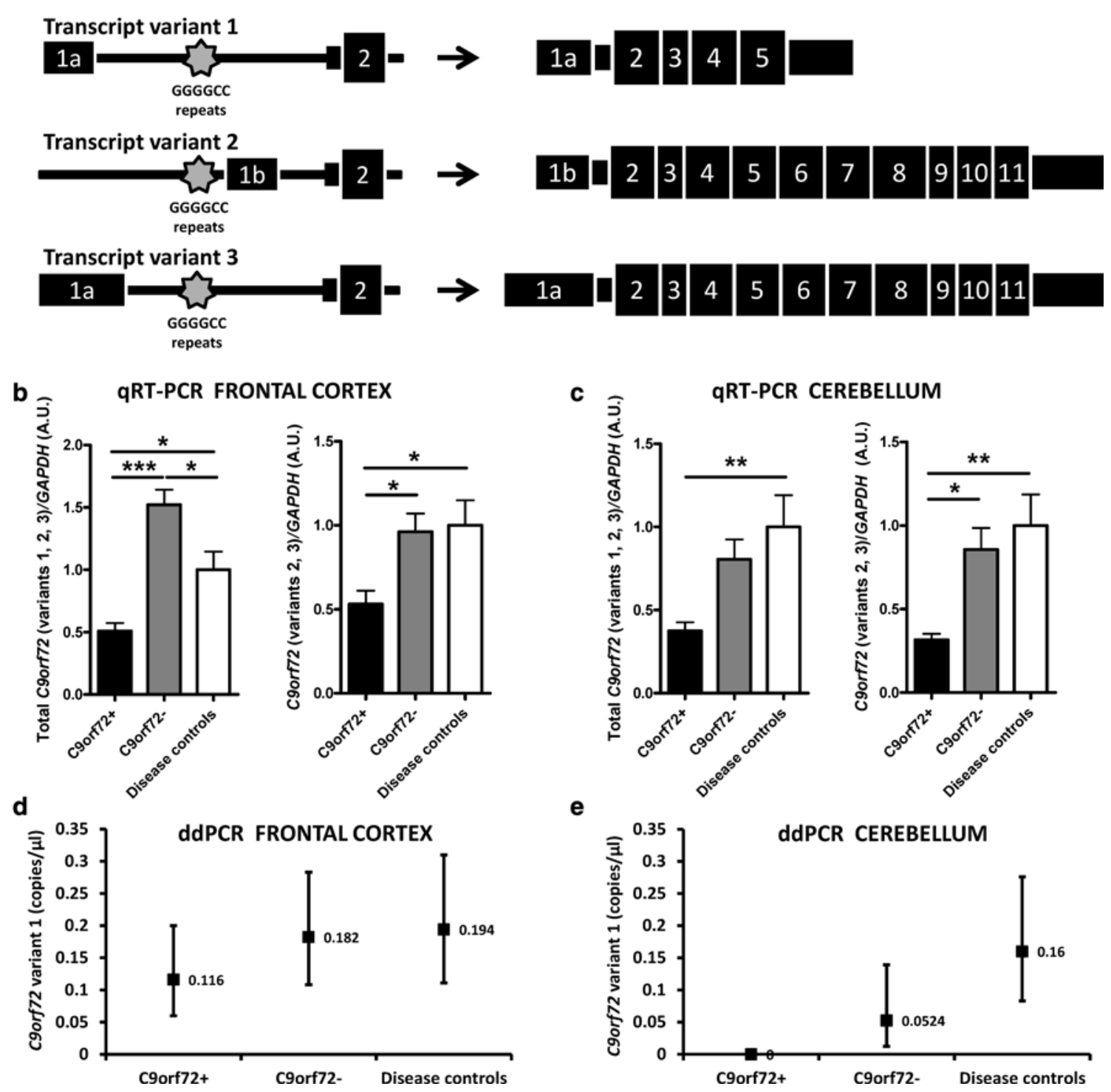

Fig. 1 Frontal cortex and cerebellum tissue samples from the C9orf72+ group exhibit a significant decrease in C9orf72 mRNA expression levels. a Schematic representation showing the three known transcript variants of C9orf72. The star marks the repeat location. b, c qRT-PCR for C9orf72 transcript variants 1, 2, 3 and C9orf72 transcript variants 2, 3 were performed in frontal cortex (b) and cerebellum (c). Data on graphs were normalized to disease control group (mean value set to 1). Statistical differences were cal-

was amplified in all samples precipitated by both anti-(pan) $\mathrm{H} 3$ and anti-(pan)H4 (complete results in online resource, Fig. 2), confirming normal C9orf72 binding to histones $\mathrm{H} 3$ and $\mathrm{H} 4$.

To confirm that reduced mRNA expression results from aberrant binding of expanded repeats to trimethylated lysine residues in c9FTD/ALS, we treated fibroblasts obtained from seven participants carrying normal alleles (C9orf72-) and seven patients carrying a (GGGGCC)

culated by one-way ANOVA with Tukey post hoc test. ${ }^{*} p<0.05$, $* * p<0.01, * * * p<0.005$. d, e ddPCR was used to calculate absolute levels of the less abundant $C 9$ orf 72 transcript variant 1 in frontal cortex (d) and cerebellum (e). Transcript expression is expressed as number of copies per microliter. The mean expression and the range of expression across all samples tested are shown for each group. A clinical description of participants is available in Table 2 (online resource)

repeat expansion $(\mathrm{C} 9$ orf72+) (clinical information in online resource, Table 3) with 5-aza-2-deoxycytidine (5-AZA), a well-known DNA and histone demethylating agent $[31,33]$. Following RNA isolation and reverse transcription, we conducted expression assays by qRTPCR using probes targeting C9orf72 transcript variants 1 , 2 and 3, as well as transcript variants 2 and 3. Following 5-AZA treatment, C9orf72 mRNA expression was significantly increased in fibroblasts from (GGGGCC) 

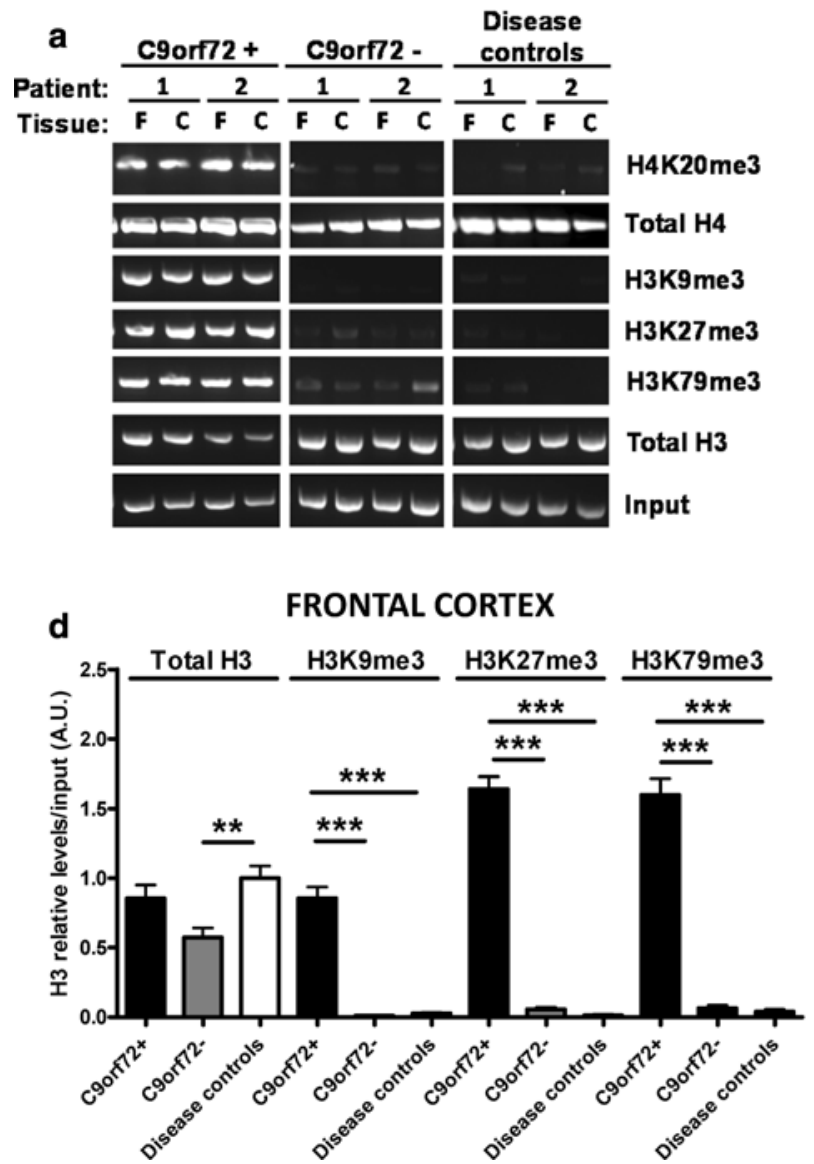

Fig. 2 Reduced $C 9$ orf 72 mRNA expression levels in the C9orf72+ group result from aberrant binding to trimethylated histone residues. a Electrophoretic representation of chromatin immunoprecipitated DNA in a subgroup of participants tested. Chromatin immunoprecipitation (ChIP) was performed on two different human tissues, frontal cortex $(\mathrm{F})$ and cerebellum $(\mathrm{C})$, using antibodies specific for total histones $\mathrm{H} 3$ and $\mathrm{H} 4$ or trimethylated histones $\mathrm{H} 3 \mathrm{~K} 9, \mathrm{H} 3 \mathrm{~K} 27$, H3K79, and H4K20. Following pull-down, bound DNA was purified and used for PCR amplification of the C9orf72 promoter region. This region was successfully amplified in the C9orf $72+$ group when ChIP was carried out with antibodies targeting trimethylated histone

repeat expansion carriers, an effect not observed in fibroblasts from participants carrying normal alleles (Fig. 3a, b). However, mRNA expression of H19, an imprinted gene, was increased in both pathological and normal repeat carriers following 5-AZA treatment (Fig. 3c). Consistent with our brain ChIP experiments, we found that H3K9me3 strongly binds to C9orf72 repeat expansions in DMSO-treated C9orf72+ fibroblasts but not in C9orf72fibroblasts. Of importance, the 5-AZA-induced increase in C9orf72 mRNA expression in C9orf72+ fibroblasts was accompanied by a decrease in binding of the $C 9$ orf 72 promoter to H3K9me3 (Fig. 3d, e). Similar to our studies using brain tissue, we found normal binding of $C 9$ orf 72 to histone $\mathrm{H} 3$ in all fibroblasts after precipitating samples
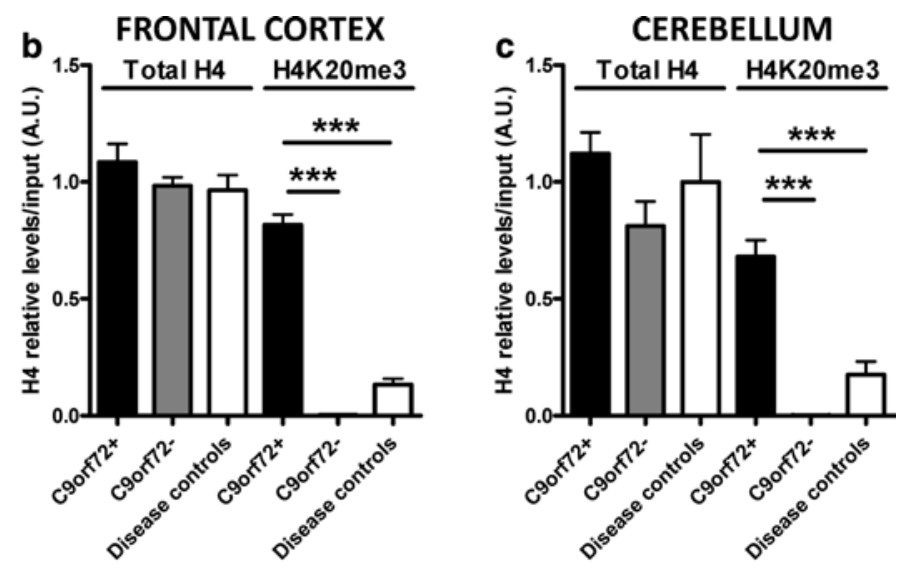

e

\section{CEREBELLUM}

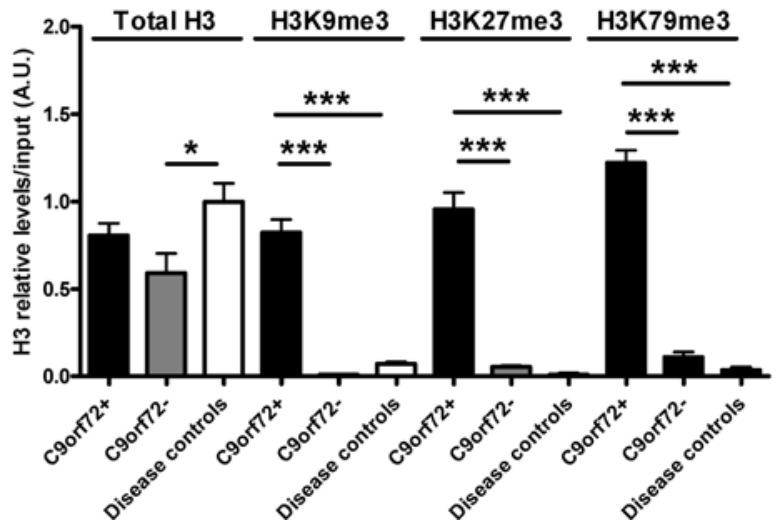

residues; under the same conditions, this region was not amplified in C9orf72- and disease controls, indicating an absence of binding. The complete figure is provided in the online resource. b, c, d, e Relative quantifications of all brain DNA were performed by measuring band intensity (complete gels in the online resource, Fig. 2) for each immunoprecipitated histone and presented as a ratio to the input. Each graph is normalized to total histone levels in the disease control group (mean value set to 1 ). Statistical differences were calculated by one-way ANOVA with Tukey post hoc test. $* \mathrm{p}<0.05$, ** $\mathrm{p}<0.01$, $* * * \mathrm{p}<0.005$. A clinical description of participants is available in Table 2 (online resource)

with anti-(pan)H3 (Fig. 3f) (complete results in online resource, Fig. 3).

Finally, to determine whether these biological changes are detectable in peripheral blood, we isolated mononuclear cells from blood collected from two repeat expansion carriers $(\mathrm{C} 9$ orf $72+)$ and two participants carrying normal alleles (C9orf72-), and conducted ChIP experiments using anti-H3K9me3, anti-H3K27me3, and anti-(pan)H3. As in the brain, we confirmed that only mutant $C 9$ orf72 strongly binds $\mathrm{H} 3 \mathrm{~K} 9 \mathrm{me} 3$ and $\mathrm{H} 3 \mathrm{~K} 27 \mathrm{me} 3$, despite normal binding of the C9orf72 promoter region to histone $\mathrm{H} 3$ in all four samples (Fig. 4).

Our experiments provide compelling evidence that repression of the C9orf72 gene in pathogenic (GGGGCC) 
a

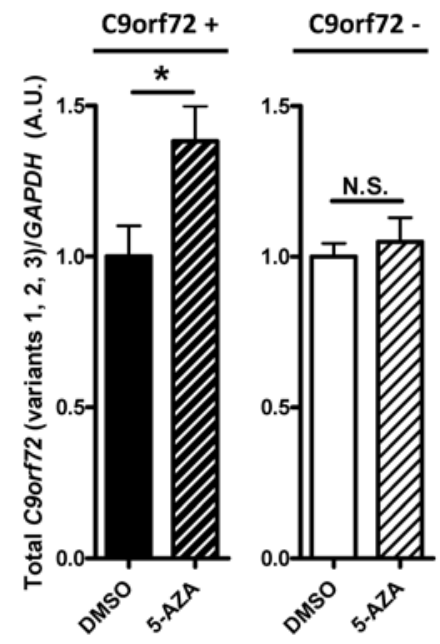

d

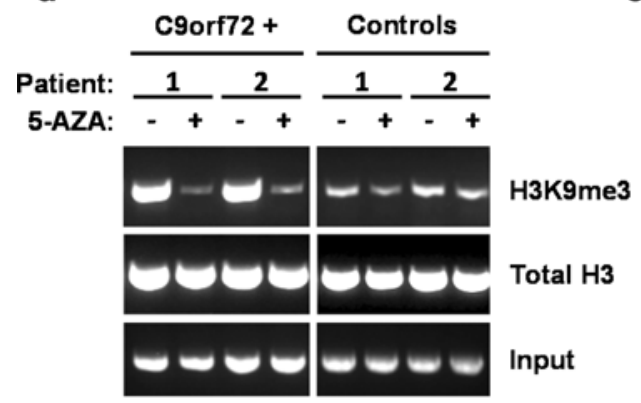

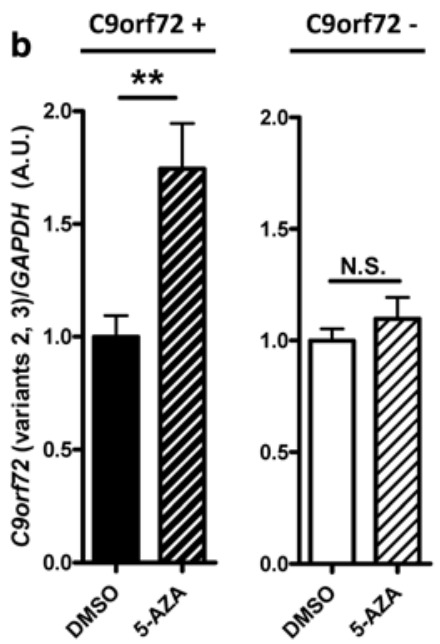

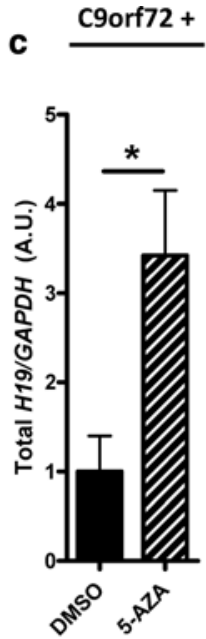

C9orf72 -

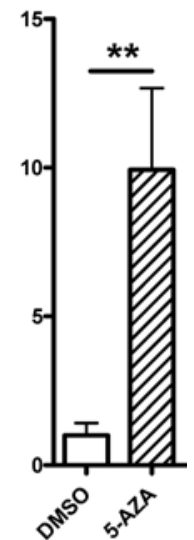

f

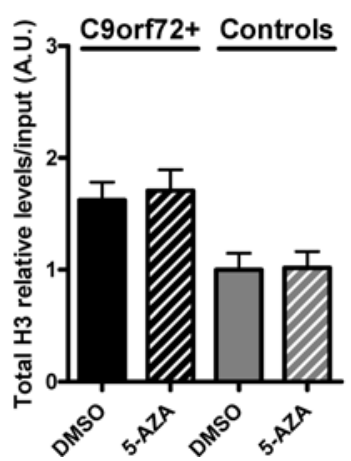

Fig. 3 C9orf72 mRNA expression is increased and H3K9me3 binding is decreased in C9orf72+ fibroblasts upon 5-AZA treatment. a, b qRT-PCR of RNA obtained from human fibroblasts grown in DMSO or 5-AZA demethylating agent. Both assays targeting transcript variants 1, 2, 3 (a) and 2, 3 (b) show a significant increase in expression after 5-AZA treatment only in $C 9$ orf 72 repeat expansion carriers. c qRT-PCR of H19, an imprinted gene, showing effectiveness of the 5-AZA treatment in C9orf72+ and C9orf72- fibroblasts. Statistical differences were assessed by unpaired Student $t$ test. ${ }^{*} p<0.05$, $* * p<0.01$. NS no significant difference. d Electrophoretic representation of chromatin immunoprecipitated DNA from a fibroblast subgroup using antibodies specific for total $\mathrm{H} 3$ or trimethylated histone H3K9. Fibroblasts were grown in DMSO or 5-AZA. Chromatin immunoprecipitation (ChIP) was performed in fibroblasts from C9orf72+ and C9orf72- participants. Following pull-down, bound

repeat carriers is caused by the binding of mutant $C 9$ orf 72 to trimethylated lysine residues within histones $\mathrm{H} 3$ and $\mathrm{H} 4$.

\section{Discussion}

In this study, we show that the decrease in C9orf72 expression levels in brain tissue and fibroblasts derived from pathogenic C9orf72 expansion carriers is associated with enhanced binding of mutant $\mathrm{C} 9$ orf72 to trimethylated lysine residues within histones $\mathrm{H} 3$ and $\mathrm{H} 4$. We also demonstrate that these epigenetic changes are detectable in
DNA was purified and used for PCR amplification of the C9orf72 promoter region. Upon treatment with vehicle (DMSO), the binding to trimethylated histone $\mathrm{H} 3 \mathrm{~K} 9$ in C9orf72+ cells, as assessed by the level of amplified $C 9$ orf 72 promoter region, was higher as compared to C9orf72-. Treatment with 5-AZA reduced this binding in C9orf72+ cases. The complete figure of all fibroblast lines is provided in the online resource. $\mathbf{e}$, $\mathbf{f}$ Relative quantifications of all fibroblast lines were performed by measuring band intensity (complete gels in the online resource, Fig. 3) for each immunoprecipitated histone and presented as a ratio to the input. Graphs are normalized to total histone levels of disease control group (mean value set to 1). Statistical differences were calculated by one-way ANOVA with Tukey post hoc test. $* p<0.05$. A clinical description of participants is available in Table 3 (online resource)

peripheral blood, a finding that, when confirmed in a larger cohort of patients, may represent a disease-specific biomarker of c9FTD/ALS.

Our data suggest that the decrease in C9orf72 mRNA expression results from heterochromatin formation following aberrant histone methylation. As C9orf72 expansions are mostly inherited in an autosomal dominant fashion, heterozygous $C 9$ orf 72 expanded repeat carriers carry both a wild-type transcriptionally active allele (euchromatin), as well as a mutated transcriptionally silent allele (heterochromatin). The wild-type allele is most likely unable to produce enough C9orf72 protein to compensate for the loss of 


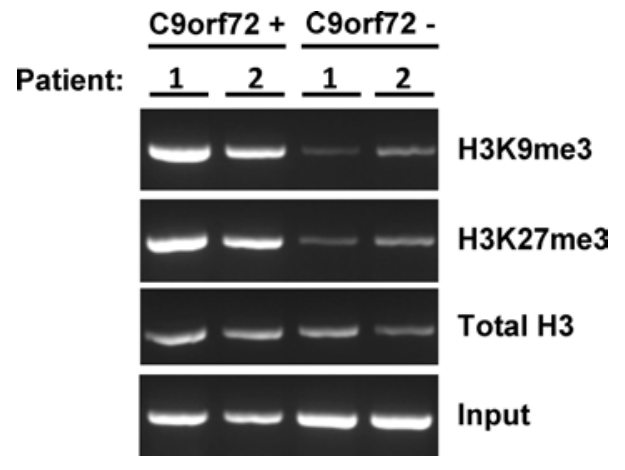

Fig. 4 Strong binding of expanded C9orf 72 to H3K9me3 and $\mathrm{H} 3 \mathrm{~K} 27 \mathrm{me} 3$ is detectable in the blood of c9FTD/ALS patients. Electrophoretic representation of chromatin immunoprecipitated blood DNA from two repeat expansion carriers (C9orf72+) and two participants carrying normal alleles (C9orf72-). Antibodies specific for total $\mathrm{H} 3$ or trimethylated histone $\mathrm{H} 3 \mathrm{~K} 9$ and $\mathrm{H} 3 \mathrm{~K} 27$ were used to pull-down DNA. Following pull-down, bound DNA was purified and used for PCR amplification of the C9orf72 promoter region. The binding to trimethylated $\mathrm{H} 3 \mathrm{~K} 9$ and $\mathrm{H} 3 \mathrm{~K} 27$ in $\mathrm{C} 9$ orf $72+$ blood cells, as assessed by the level of amplified $C 9$ orf 72 promoter region, was higher as compared to C9orf72-

protein from the mutated allele, thereby leading to haploinsufficiency (Fig. 5). As histone methylation is commonly perceived as inducing flexible short-term gene silencing through regulation of numerous histone-modifying complexes, regulation of the chromatin structure varies depending on the availability of chromatin remodeling enzymes, including histone acetyltransferases, histone deacetylases, histone methyltransferases and histone demethylases. This dynamic chromatin structure likely influences transcription of expanded $C 9$ orf 72 , which consequently might either accumulate as RNA foci or translate into aggregation-prone c9RAN proteins.

While our studies were in progress, the Rogaeva's group reported DNA hypermethylation of the upstream $\mathrm{CpG}$ island in about $40 \%$ of all ALS cases that were tested [43]. Although they found no hypermethylation in about $60 \%$ of the ALS cases studied, it remains possible that another $\mathrm{CpG}$ island located farther away from the repeat might be methylated in these patients. Several $\mathrm{CpG}$ sites within the repeat itself may also be methylated, as suggested by the methyl-sensitive restriction enzyme digest and sequencing (MRE-seq) $\mathrm{CpG}$ score tool (http://genome.ucsc.edu). Of note, the number of $\mathrm{CpG}$ sites in the repeat region is increased in expansion carriers, creating a new $\mathrm{CpG}$ island that can be a target for methylation. Future studies will need to determine whether the expanded repeat as well as nearby $\mathrm{CpG}$ islands are methylated, and estimate the frequency of aberrant DNA methylation in both ALS and FTD cases. While DNA methylation is commonly believed to trigger histone methylation, we now know that methylation of either DNA or histone residues can appear first, leading to aberrant methylation of the other structure [10]. If DNA methylation appears first, it must happen well before the onset of symptoms; increased binding of mutant $\mathrm{C} 9$ orf 72 to trimethylated histone $\mathrm{H} 3 \mathrm{~K} 9$ was similarly present in two asymptomatic repeat carriers (28 and 30 years of age) and in patients already diagnosed with ALS or FTD (clinical information in online resource, Table 3). The expanded repeat may also lead to an unusual DNA structure, increasing DNMTs' capacity to catalyze methyl groups to cytosine residues within the repeat itself. This aberrant DNA methylation in turn may trigger methylation of histones $\mathrm{H} 3$ and H4. Regardless of which structure becomes methylated first, our findings support the notion that it is the aberrant methylation of histones $\mathrm{H} 3$ and $\mathrm{H} 4$ residues in all c9FTD/ALS patient samples evaluated that caused the decreased expression of $C 9$ orf 72 mRNA.

The expanded non-coding (GGGGCC) repeat, which is located between two alternatively spliced non-coding $5^{\prime}$ exons of $C 90 r f 72$, produces three distinct transcript

\section{HETEROZYGOUS C9orf72 EXPANDED REPEAT CARRIERS}

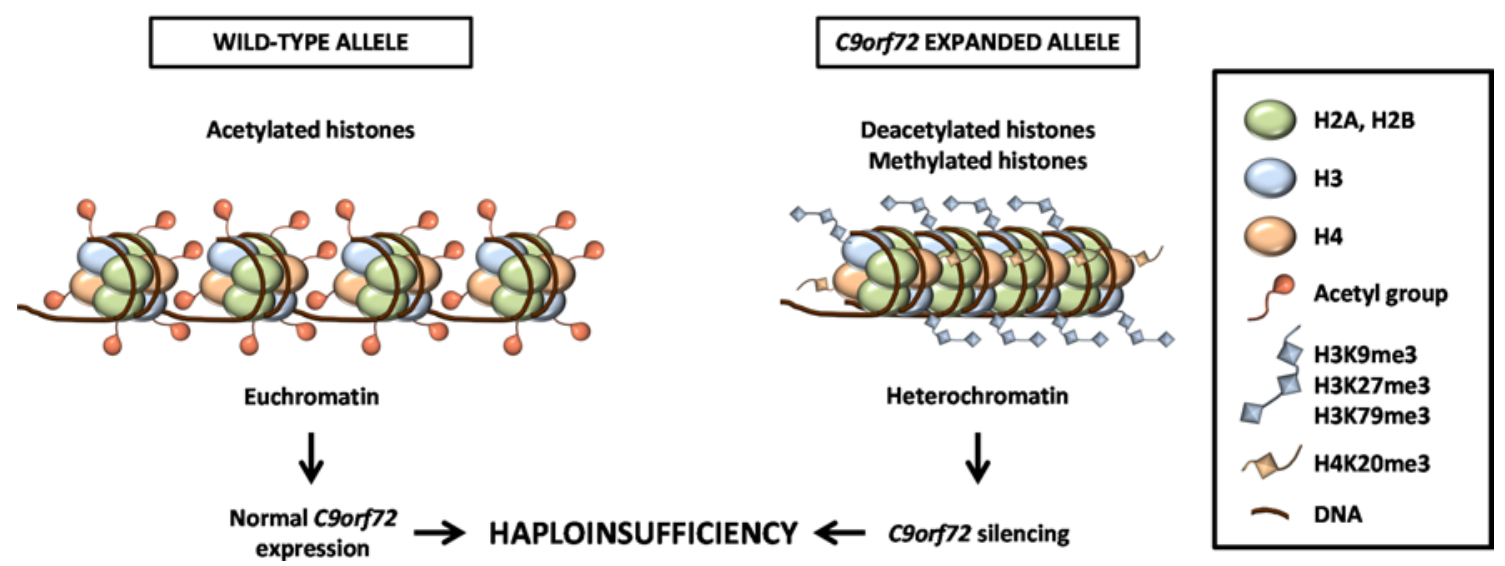

Fig. 5 Schematic representation of the proposed haploinsufficiency mechanism resulting from epigenetic changes in c9FTD/ALS 
variants (Fig. 1a). Transcript variants 1 (NM_145005.5) and 3 (NM_001256054.1) have the repeat located in the intronic region and have been suggested to lead to RNA foci formation and RNA-binding protein sequestration, while the expression of variant 2 (NM_018325.3), with the repeat located in the promoter region, has been shown to be silenced in pathogenic repeat carriers [16, 29]. Others reported reduced expression of the three known transcript variants [22]. Given this difference in repeat location and considering the involvement of epigenetic changes, the transcription level of each variant may not be uniformly altered by methylation, thereby resulting in different disease courses and/or biological outcomes. Such methylation variability has previously been shown to strongly influence phenotypes in Fragile X and Rett syndromes [8, 40]. Future studies will need to evaluate whether methylation variability similarly influence ALS and FTD phenotypes.

Our results rise important questions regarding the mid-adulthood onset of disease, as the methylation pattern of histones $\mathrm{H} 3$ and $\mathrm{H} 4$ varies with age, increasing or decreasing depending on histone residues [11]. Of interest, the trimethylated histone residues aberrantly bound by mutant $C 9$ orf 72 are also known to be methylated in an age-dependent manner. Specifically, methylation at residue H3K9 decreases with age, and methylation at residues H3K27, H3K79 and H4K20 increases [17, 38, 42]. Moreover, it is well-known that accumulation of reactive oxygen species over time leads to an exponential increase in molecular oxidative stress, an event extensively associated with ALS [12], which has been suggested to cause a gradual decline in homeostatic mechanisms associated with aging [5, 39]. It has also been suggested that epigenetic mechanisms resulting from aging possibly play an important physiopathological role in the presence of oxidative stress [11]. Oxidative stress is known to alter the balance between acetylation/deacetylation and methylation/demethylation processes in histone residues, deregulating the expression of pro-inflammatory genes. As such, aberrant histone methylation as a result of $C 9$ orf 72 repeat expansions, oxidative stress accumulation leading to epigenetic deregulation, and normal age-related epigenetic mechanism variations could all contribute to the adult onset of the disease as well as the neuroinflammation found in ALS patients [44]. Since c9FTD/ALS histone-associated methylation can be easily detected using patient blood, it will be of interest to evaluate whether aberrant histone methylation changes over the course of the disease.

Our results suggest that c9FTD/ALS can now be considered among the class of repeat disorders marked by chromatin modifications, such as myotonic dystrophy, SCA types 8 and 31, Friedreich ataxia, Fragile $\mathrm{X}$ and Fragile $\mathrm{X}$-associated tremor/ataxia syndromes [6]. Appropriate chromatin conformation in neurons is essential to prevent DNA damage, to assure proper DNA packaging and to maintain normal gene expression. Similar to myotonic dystrophy and SCA8, three distinct pathogenic processes appear to be associated with c9FTD/ALS pathogenesis. First, C9orf72 repeat expansions lead to the formation of RNA foci, which have the potential to sequester RNA-binding proteins and impair their function [16, 29]. Second, we and others have recently reported that $C 9$ orf 72 repeat expansions lead to RAN translation resulting in the production of poly-glycine-alanine, poly-glycine-arginine and poly-glycine-proline peptides that accumulate as insoluble aggregates in the cytoplasm of neurons $[2,30]$, further supporting an RNAmediated mechanism of toxicity in c9FTD/ALS. Third, our current study suggests that binding of mutant $C 9$ orf 72 to trimethylated lysine residues within histones $\mathrm{H} 3$ and $\mathrm{H} 4$ causes repression of the $C 9$ orf 72 gene, an event that may play an important role in the development of c9FTD/ALS. Taken together, early studies suggest a C9orf72 loss of function, both through neurotoxic processes and epigenetic changes, as a disease mechanism, and demonstrate that a better understanding of the C9orf72 protein function is crucial in order to identify therapeutic targets and to develop effective treatment strategies [21]. Additional research to examine DNA and histone methylation as a part of c9FTD/ALS pathogenesis may also lead to the identification of novel therapeutic targets for ALS and FTD. The need for such work is urgent: despite an enormous effort to elucidate the molecular mechanisms involved in FTD and ALS, the prognosis for patients has not improved, and no effective treatment has been developed. The discovery that C9orf72 haploinsufficiency contributes to disease pathogenesis and that epigenetic processes mediate this effect offers a unique opportunity for therapeutic development, providing c9FTD/ALS patients with new hope.

Acknowledgments We would like to thank the patients involved in this study as well as acknowledge the technical support of Jeannette N. Stankowski, Yong-Jie Zhang, Karen R. Jansen-West, Jennifer M. Gass, Kristin Staggs-Douberly, Pamela Desaro, Amelia Johnston, Marka M. Van Blitterswijk, Patricia H. Brown, Melissa E. Murray, Kevin F. Bieniek, Amanda M. Liesinger, and Linda G. Rousseau from Mayo Clinic. This work was supported by Mayo Clinic Foundation, National Institutes of Health/National Institute on Aging [R01AG026251 (LP)], National Institutes of Health/National Institute of Neurological Disorders and Stroke [R01 NS 063964-01 (LP), R01 NS077402 (LP)], National Institute of Environmental Health Services [ES20395-01 (LP)], Amyotrophic Lateral Sclerosis Association (LP), the Canadian Institutes of Health Research (VVB), and the Siragusa Foundation (VVB).

Conflict of interest The authors declare that they have no conflict of interest.

Open Access This article is distributed under the terms of the Creative Commons Attribution License which permits any use, distribution, and reproduction in any medium, provided the original author(s) and the source are credited. 


\section{References}

1. Al-Mahdawi S, Pinto RM, Ismail O, Varshney D, Lymperi S, Sandi C et al (2008) The Friedreich ataxia GAA repeat expansion mutation induces comparable epigenetic changes in human and transgenic mouse brain and heart tissues. Hum Mol Genet 17(5):735-746

2. Ash PE, Bieniek KF, Gendron TF, Caulfield T, Lin WL, Dejesus-Hernandez $M$ et al (2013) Unconventional translation of C9ORF72 GGGGCC expansion generates insoluble polypeptides specific to c9FTD/ALS. Neuron 77(4):639-646

3. Barski A, Cuddapah S, Cui K, Roh TY, Schones DE, Wang Z et al (2007) High-resolution profiling of histone methylations in the human genome. Cell 129(4):823-837

4. Bauer PO, Zumrova A, Matoska V, Mitsui K, Goetz P (2004) Can ataxin-2 be down-regulated by allele-specific de novo DNA methylation in SCA2 patients? Med Hypotheses 63(6):1018-1023

5. Beckman KB, Ames BN (1998) The free radical theory of aging matures. Physiol Rev 78(2):547-581

6. Belzil VV, Gendron TF, Petrucelli L (2012) RNA-mediated toxicity in neurodegenerative disease. Mol Cell Neurosci 56C:406-419

7. Bradley WG (2000) Neurology in clinical practice, 3rd edn. Butterworth-Heinemann, Boston

8. Braunschweig D, Simcox T, Samaco RC, LaSalle JM (2004) $\mathrm{X}$-Chromosome inactivation ratios affect wild-type MeCP2 expression within mosaic Rett syndrome and Mecp2干 mouse brain. Hum Mol Genet 13(12):1275-1286

9. Caiafa P, Zampieri M (2005) DNA methylation and chromatin structure: the puzzling $\mathrm{CpG}$ islands. J Cell Biochem 94(2):257-265

10. Cedar H, Bergman Y (2009) Linking DNA methylation and histone modification: patterns and paradigms. Nat Rev Genet 10(5):295-304

11. Cencioni C, Spallotta F, Martelli F, Valente S, Mai A, Zeiher AM et al (2013) Oxidative stress and epigenetic regulation in ageing and age-related diseases. Int J Mol Sci 14(9):17643-17663

12. Chen S, Sayana P, Zhang X, Le W (2013) Genetics of amyotrophic lateral sclerosis: an update. Mol Neurodegener 8(1):28

13. Chestnut BA, Chang Q, Price A, Lesuisse C, Wong M, Martin LJ (2011) Epigenetic regulation of motor neuron cell death through DNA methylation. J Neurosci 31(46):16619-16636

14. Ciura S, Lattante S, Le Ber I, Latouche M, Tostivint H, Brice A et al (2013) Loss of function of C9orf72 causes motor deficits in a zebrafish model of amyotrophic lateral sclerosis. Ann Neurol. doi:10.1002/ana.23946

15. Cong SY, Pepers BA, Zhou TT, Kerkdijk H, Roos RA, van Ommen GJ et al (2012) Huntingtin with an expanded polyglutamine repeat affects the Jab1-p27(Kip1) pathway. Neurobiol Dis 46(3):673-681

16. DeJesus-Hernandez M, Mackenzie IR, Boeve BF, Boxer AL, Baker M, Rutherford NJ et al (2011) Expanded GGGGCC hexanucleotide repeat in noncoding region of C9ORF72 causes chromosome 9p-linked FTD and ALS. Neuron 72(2):245-256

17. Fraga MF, Esteller M (2007) Epigenetics and aging: the targets and the marks. Trends Genet 23(8):413-418

18. Galimberti D, D'Addario C, Dell'osso B, Fenoglio C, Marcone A, Cerami C et al (2013) Progranulin gene (GRN) promoter methylation is increased in patients with sporadic frontotemporal lobar degeneration. Neurol Sci 34(6):899-903

19. Galimberti D, Scarpini E (2010) Genetics and biology of Alzheimer's disease and frontotemporal lobar degeneration. Int J Clin Exp Med 3(2):129-143

20. Gardiner-Garden M, Frommer M (1987) $\mathrm{CpG}$ islands in vertebrate genomes. J Mol Biol 196(2):261-282
21. Gendron TF, Cosio DM, Petrucelli L (2013) c9RAN translation: a potential therapeutic target for the treatment of amyotrophic lateral sclerosis and frontotemporal dementia. Expert Opin Ther Targets 17(9):991-995

22. Gijselinck I, Van Langenhove T, van der Zee J, Sleegers K, Philtjens S, Kleinberger G et al (2012) A C9orf72 promoter repeat expansion in a Flanders-Belgian cohort with disorders of the frontotemporal lobar degeneration-amyotrophic lateral sclerosis spectrum: a gene identification study. Lancet Neurol 11(1):54-65

23. Giordana MT, Ferrero P, Grifoni S, Pellerino A, Naldi A, Montuschi A (2011) Dementia and cognitive impairment in amyotrophic lateral sclerosis: a review. Neurol Sci 32(1):9-16

24. Graff-Radford NR, Woodruff BK (2007) Frontotemporal dementia. Semin Neurol 27(1):48-57

25. Hindson CM, Chevillet JR, Briggs HA, Gallichotte EN, Ruf IK, Hindson BJ et al (2013) Absolute quantification by droplet digital PCR versus analog real-time PCR. Nat Methods 10(10):1003-1005

26. Johnston CA, Stanton BR, Turner MR, Gray R, Blunt AH, Butt D et al (2006) Amyotrophic lateral sclerosis in an urban setting: a population based study of inner city London. J Neurol 253(12):1642-1643

27. Laffita-Mesa JM, Bauer PO, Kouri V, Pena Serrano L, Roskams J, Almaguer Gotay D et al (2012) Epigenetics DNA methylation in the core ataxin-2 gene promoter: novel physiological and pathological implications. Hum Genet 131(4):625-638

28. Lomen-Hoerth C, Anderson T, Miller B (2002) The overlap of amyotrophic lateral sclerosis and frontotemporal dementia. Neurology 59(7):1077-1079

29. Mori K, Lammich S, Mackenzie IR, Forne I, Zilow S, Kretzschmar $\mathrm{H}$ et al (2013) hnRNP A3 binds to GGGGCC repeats and is a constituent of p62-positive/TDP43-negative inclusions in the hippocampus of patients with C9orf72 mutations. Acta Neuropathol 125(3):413-423

30. Mori K, Weng SM, Arzberger T, May S, Rentzsch K, Kremmer E et al (2013) The C9orf72 GGGGCC repeat is translated into aggregating dipeptide-repeat proteins in FTLD/ALS. Science 339(6125): $1335-1338$

31. Murgo AJ (2005) Innovative approaches to the clinical development of DNA methylation inhibitors as epigenetic remodeling drugs. Semin Oncol 32(5):458-464

32. Nasrallah MP, Cho G, Simonet JC, Putt ME, Kitamura K, Golden JA (2012) Differential effects of a polyalanine tract expansion in Arx on neural development and gene expression. Hum Mol Genet 21(5):1090-1098

33. Oki Y, Issa JP (2006) Review: recent clinical trials in epigenetic therapy. Rev Recent Clin Trials 1(2):169-182

34. Prell T, Grosskreutz J (2013) The involvement of the cerebellum in amyotrophic lateral sclerosis. Amyotroph Lateral Scler Frontotemporal Degener (Epub ahead of print)

35. Renoux AJ, Todd PK (2012) Neurodegeneration the RNA way. Prog Neurobiol 97(2):173-189

36. Renton AE, Majounie E, Waite A, Simon-Sanchez J, Rollinson S, Gibbs JR et al (2011) A hexanucleotide repeat expansion in C9ORF72 is the cause of chromosome 9p21-linked ALS-FTD. Neuron 72(2):257-268

37. Santoro MR, Bray SM, Warren ST (2012) Molecular mechanisms of fragile X syndrome: a twenty-year perspective. Annu Rev Pathol 7:219-245

38. Sarg B, Koutzamani E, Helliger W, Rundquist I, Lindner HH (2002) Postsynthetic trimethylation of histone $\mathrm{H} 4$ at lysine 20 in mammalian tissues is associated with aging. J Biol Chem 277(42):39195-39201

39. Stadtman ER (1992) Protein oxidation and aging. Science 257(5074):1220-1224 
40. Stoger R, Kajimura TM, Brown WT, Laird CD (1997) Epigenetic variation illustrated by DNA methylation patterns of the fragile-X gene FMR1. Hum Mol Genet 6(11):1791-1801

41. Todd PK, Oh SY, Krans A, Pandey UB, Di Prospero NA, Min KT et al (2010) Histone deacetylases suppress CGG repeat-induced neurodegeneration via transcriptional silencing in models of fragile $\mathrm{X}$ tremor ataxia syndrome. PLoS Genet 6(12):e1001240

42. Wang CM, Tsai SN, Yew TW, Kwan YW, Ngai SM (2010) Identification of histone methylation multiplicities patterns in the brain of senescence-accelerated prone mouse 8 . Biogerontology 11(1):87-102

43. Xi Z, Zinman L, Moreno D, Schymick J, Liang Y, Sato C et al (2013) Hypermethylation of the $\mathrm{CpG}$ island near the GC repeat in ALS with a C9orf72 expansion. Am J Hum Genet (Epub ahead of print)

44. Zhao W, Beers DR, Appel SH (2013) Immune-mediated mechanisms in the pathoprogression of amyotrophic lateral sclerosis. $\mathrm{J}$ Neuroimmune Pharmacol 8(4):888-899 